\title{
Erratum to: Male Age Affects Female Mate Preference and Reproductive Performance in the Cabbage Beetle, Colaphellus bowringi
}

\author{
Xing-Ping Liu • Jing Xu • Hai-Min He • \\ Xian-Ju Kuang • Fang-Sen Xue
}

The original version of this article unfortunately contained a mistake on the acknowledgements. The number of the project supported our research is 31000173 , instead of 31000176 .

The online version of the original article can be found at http://dx.doi.org/10.1007/s10905-010-9237-5.

X.-P. Liu • J. Xu • H.-M. He • X.-J. Kuang • F.-S. Xue $(\bowtie)$

Institute of Entomology, Jiangxi Agricultural University, Nanchang 330045, China

e-mail: xue_fangsen@hotmail.com

X.-P. Liu

e-mail: jxaulxp@163.com 